(c) 2010 IEEE. Personal use of this material is permitted. Permission from IEEE must be obtained for all other uses, in any current or future media, including reprinting/republishing this material for advertising or promotional purposes, creating new collective works, for resale or redistribution to servers or lists, or reuse of any copyrighted component of this work in other works. 


\section{Prototype Design and Analysis of Wireless Vibration Sensor}

\author{
Mohammad reza Akhondi, Alex Talevski \\ DEBII \\ Curtin University of Technology, \\ Perth, Western Australia, \\ M.Akhondi@postgrad.curtin.edu.au \\ A.Talevski@curtin.edu.au
}

\author{
Tsung-Hsien Chou \\ Curtin University of Technology \\ Perth, Western Australia \\ chou1332@hotmail.com
}

\begin{abstract}
Since the technology era began, sensor systems have been frequently updated. In order to keep the sensor up to date, components are designed using a modular approach that can be updated with the latest technology. The objective of this paper is to illustrate the design of a vibration sensor that integrates an enhanced Mechanism for Hardware Health Monitoring (MHHM). The design achieves the digitizing, wireless communication and modularity aspects of this system as required. The selection of appropriate components is also presented in the paper as a guide.
\end{abstract}

Keywords-component; vibration sensor; Mechanical hardware health monitoring, wireless communication

\section{INTRODUCTION}

SmartVib sensor is an analog based device which responds to vibration and preprocesses the signal in a digital format. The output data is then transmitted through a cable for detailed analysis.

The SmartVib's objective was to obtain the vibration from subjected machine. It uses the vibration result to predict the machine's health condition or lifespan. This technique is called Mechanism Hardware Health Monitoring (MHHM).

However, there are few drawbacks due to this decades old technology. Analog based device has a common disadvantage in signal processing and it is known as noise. Noise is the random unwanted variation. It causes data loss by distortion and it is difficult to be detect, isolate and rectify.

The predecessor, SmartVib, only provides two output data parameters which is amplitude and frequency. Both parameters are known as the core parameters of vibration calculation; the boosting hardware health analysis requires multiple data that should be pre-calculated by the sensor device on-the-fly.

The data transmission method used by the SmartVib is dependent on cabling. Cabling acts as a bridge that connects the sensor to personal computer. It is a one-to-one point connection. Transmission distance is one of many factors that induce lost data. Long distance transmission will have high possibility in data loses. Moreover, Cabling does not work well in hazards condition due to corrosion, aging, insulation layer damaging and etc.

According to Moore's Law [1], the technology advances exponentially. The capability of upgrading software and hardware for such instruments needs to be scrutinized. SmartVib was built seven years ago without considering modular design. It is impossible to upgrade this device. The only option was to redesign.

Last but not the least, decade old technology is physically larger in dimension compared to the current surface mount technology (SMT).

\section{GENERAL BACKGROUND INFORMATION}

This section unravels the vibration theory that is required in the software part of this research. It follows by the Fast Furious Transform (FFT) method that uses to convert collecting signal from time domain to frequency domain.

\section{A. Vibration theory}

Vibration takes a form of sinusoidal wavelike movement. The response of its displacement can be represented by a fundamental formula, $X=A \cos (\omega t)$ where $\omega=2 \pi f$ and $A=$ amplitude [2]. Differentiate the displacement equation to obtain the velocity, $v$, and the equation becomes

$\mathrm{X}=v=-A \omega \sin (\omega t)$

The next order up, acceleration (a), is basically differentiate the velocity equation. The equation now becomes

$\mathrm{X}=a=-A \omega^{2} \cos (\omega t)$

The magnitude of acceleration is Aw2.

' $\mathrm{g}$ level' also known as gravity level, is a measurement unit of vibration. One gravity level is equivalent to the acceleration of gravity, $9.81 \mathrm{~m} / \mathrm{s} 2$. A typical output from an accelerometer is $\mathrm{g}$ level. Let's make the magnitude of acceleration, $A w^{2}=g$. Then the amplitude equation becomes

$$
A=\frac{g}{\omega^{2}}=\frac{g}{(2 \pi f)^{2}}
$$

Equation (3), shows the formula to calculate the amplitude if the frequency and $g$ level is provided by the accelerometer. 


\section{B. Fault diagnosis}

Akhondi also claims that fault diagnosis is cataloged into two regions; component fault diagnosis and system fault diagnosis. This project only considers the component fault diagnosis as it is designated to test individual hardware health monitoring [3].

The vibration sensor is not limited to health monitoring. It can also be used in situation where unwanted vibration can be detected and warning issued to the monitoring system. An example is where two machines are connected together where alignment of both machines is critical. If the amplitude of vibration exceeds its acceptable boundary, it would cause both machines to be damaged which could lead to disaster.

\section{Natural frequency of a uniform beam}

A simplest form of uniform beam is a free-free beam system. There is no restriction on both ends of the beam. Alternatively, the beam is free to response to applied excitation source. In order to obtain the natural frequency of the free-free beam, Inman states that equation of characteristic natural frequency of free-free beam is shown as follow [6].

$$
\lambda^{4}=\frac{\omega^{2} A \rho}{E I}
$$

Where,

$\mathrm{E}=$ Young's Modulus

$\mathrm{I}=$ Second moment of inertia (area moment of inertia)

$\lambda=$ Wavelength

$\mathrm{A}=$ Cross section area.

$\rho=$ Density

$\omega=$ angular velocity (calculated from characteristic of root)

The parameters above are dependent on the properties of the beam.

\section{Testing hardware specification and approach}

Testing hardware is purposely prepared to create a theoretical reference value for comparison with the result from purposed sensor measurement. The following table gives the specification of the testing hardware.

Table 1: Testing hardware specification

\begin{tabular}{|c|c|c|c|}
\hline \multicolumn{4}{|c|}{ Steel vibration beam (steel, Mild 1020) } \\
\hline Parameters & Symbols & Figures & Descriptions \\
\hline $\begin{array}{c}\text { Length of } \\
\text { beam }\end{array}$ & $\mathrm{L}$ & $0.5 \mathrm{~m}$ & \\
\hline $\begin{array}{c}\text { Width of } \\
\text { beam }\end{array}$ & $\mathrm{W}_{\text {width }}$ & $0.05 \mathrm{~m}$ & \\
\hline $\begin{array}{c}\text { Thickness of } \\
\text { beam }\end{array}$ & $\mathrm{Tk}_{\text {thickness }}$ & $0.03 \mathrm{~m}$ & \\
\hline $\begin{array}{c}\text { Cross section } \\
\text { area }\end{array}$ & $\mathrm{A}$ & $0.0015 \mathrm{~m}^{2}$ & \\
\hline
\end{tabular}

\begin{tabular}{|c|c|c|c|}
\hline Density & $\rho$ & $7800\left(\mathrm{~kg} / \mathrm{m}^{3}\right)$ & {$[8]$} \\
\hline $\begin{array}{c}\text { Young's } \\
\text { Modulus }\end{array}$ & $\mathrm{E}$ & $210 \mathrm{GPa}$ & {$[8]$} \\
\hline $\begin{array}{c}\text { Second } \\
\text { moment of } \\
\text { inertia }\end{array}$ & $\mathrm{I}$ & $3.75 \times 10^{-9}$ & $\begin{array}{c}\mathrm{b}=\text { width; } \mathrm{h}=\text { height } \\
\mathrm{I}=\mathrm{bh}^{3} / 12 \\
{[8]}\end{array}$ \\
\hline
\end{tabular}

1) Testing Scenariosons

First scenario is a free-free vibration beam intentionally defined for theoretical prediction on vibration behavior. Software prediction assumes an ideal condition that excludes any disturbance or error. Another scenario is practical measurement that obtains from the WiVi sensor. Both outcomes of the scenarios are to be compared in order to criticize the WiVi sensor.

\section{2) Approach}

By using the software, Matlab, to find the mode shape of the steel beam; where the supporting mechanism of the beam is placed at the stationary point. This is to produce an accurate result by preventing the vibration propagation to supporting mechanism.

Matlab will predicts the frequency of the beam and the profile can be estimated within a define time frame. WiVi sensor can then be placed on the top of the steel beam for collecting vibration signal.

\section{DESIGN OVERVIEW}

This section is going to present the design process of this project. The design process can be depicted by the flowchart shown in Figure 1. The design is separated into 3 stages. The first stage is to come up with a mock design. This design will then be further explored in detailed. Lastly, a testing method will be written up for once the prototype is ready for a test run.

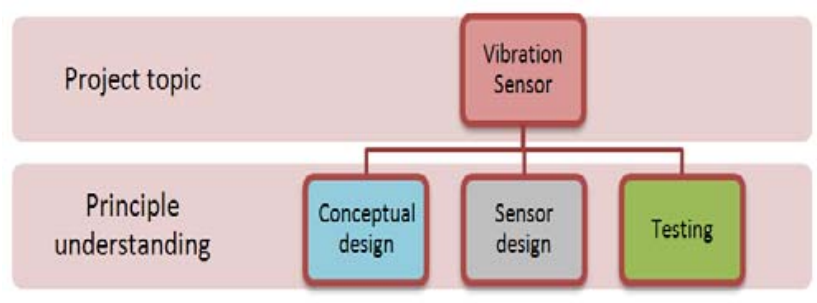

Figure1: Design concept flow chart

Conceptual design is mainly focused on the principle of theoretical knowledge that gives relevant information to organize sensor hardware. Conceptual design also states the requirements to narrow down the vast selection options. Testing is to demonstrate the proposed vibration sensor on its mechanism hardware health monitoring system. 


\section{A. Conceptual design}

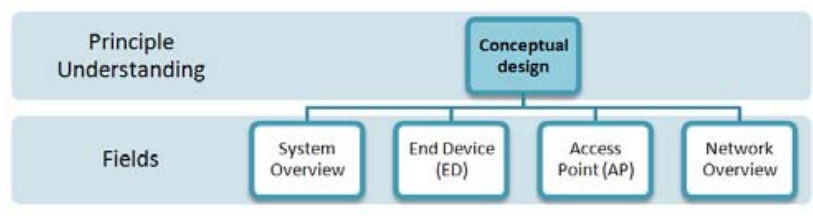

Figure2: Conceptual design section

Firstly, conceptual design can be further divided into four major areas; System overview, end device, access point, and network overview. System overview explores the system as a whole. It provides the big picture of the system where the sensor reacts to the surrounding hardware such as the PC. Next, the end device (ED) which is part of the sensor will be described. Access point (AP) is the bridge between the PC and end device. Lastly, the network within the system will be clarified.

1) System overview

There are a few requirements that need to be met by this system. The know requirements can be listed as follows.

- Sensor needs to communicate wirelessly

- Sensor must be connected to PC where the user can acquire the data

- Sensor should be modular, meaning it can be

- removed out of this system and be replaced by

- another similar sensor with better equipments.

- Sensors should be multiple-to-one relation with the PC

Before the project can start, a concept based design must be prepared. Taking the requirement above into account, the overview of the system is shown by Figure 3 .

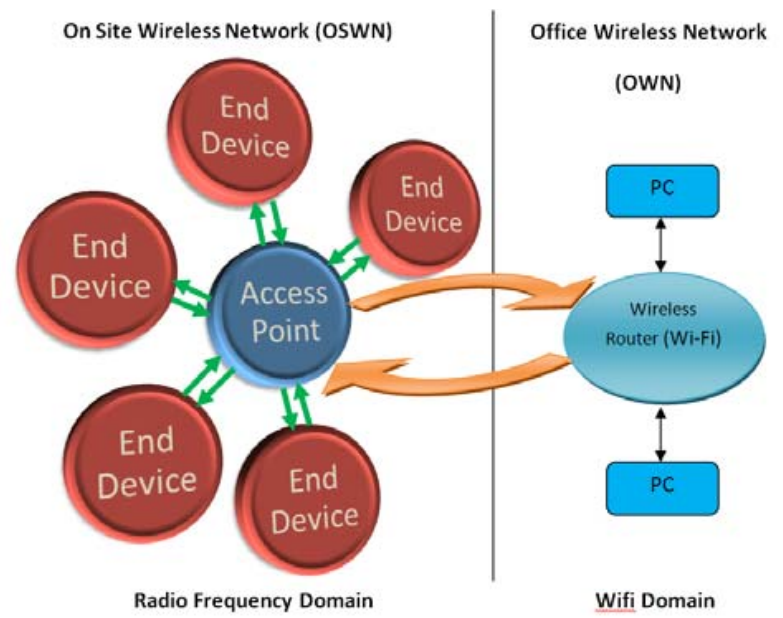

Figure 3: Ideal mechanism hardware health monitoring system
The vibration monitoring system operates in two network domains. They are On Site Wireless Network (OSWN) and Office Wireless Network (OWN). OSWN includes the access point and end device. End device represents the vibration sensor which is responsible for detecting mechanism vibration behavior. Access point represents the wireless access point between end device and wireless router.

As shown, the system contains multiple ED. It means that the user can connect more than one sensor at a time. These sensors communicate in radio frequency with the AP. The $\mathrm{AP}$ will then connect wirelessly with a generic router which in turns sends the information to a PC. The communication between AP and router has a various wireless options.

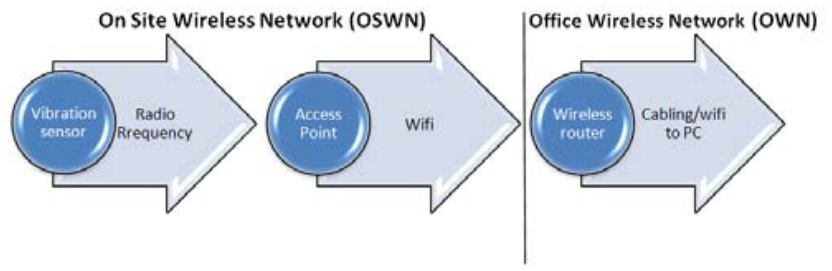

Figure4: Data transmission in signal phase.

\section{2) End device}

End Device (ED) is the actual sensor. This part of the design is the crown jewel of the project. The sensor also has multiple limitations bounded to its design. They are listed below.

- Sensor output must be digital

- $\quad$ Sensor must be modular with parts on-board

- Sensor wireless communication in radio frequency

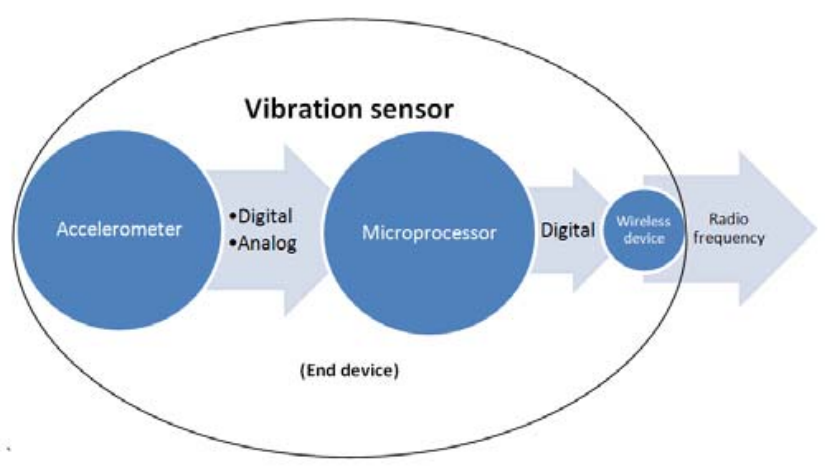

Figure5: Schematic diagram of end device.

The diagram above, Figure 5, is presenting the physical configuration of an end device. Basically, the idea of ED is divided into three different parts. Each part has its individual function in contribution to the sensor. The responsibility of each part will be explained individually by the following sub-chapters. 


\section{a) Accelerometer}

Accelerometer is responsible for vibration detection. In general, accelerometer converts the real world energy or mechanical energy, into signal. The output data can be analog or digital. The data will be transmitted to a microcontroller through a hard connection. The specification of the physical connection will be detailed in sensor design [9][10].

\section{b) Microcontroller}

Microcontroller is used to collect and manipulate the input data from the accelerometer. The microcontroller is the center of the sensor; it takes the information from the accelerometer, process the data, and sends them wirelessly to the AP. The software pre-programmed in the microcontroller will execute the above procedure accordingly.

\section{c) Wireless}

Wireless communication is particularly to form a connection route from one electronic device to another. This part can be performed by an off-shelves product. The electric signal converts into receivable radio frequency and transmit within OSWN.

\section{d) Access point}

The access point can be represented by Figure 6. As shown, it can also be represented by a few parts just like an end device.

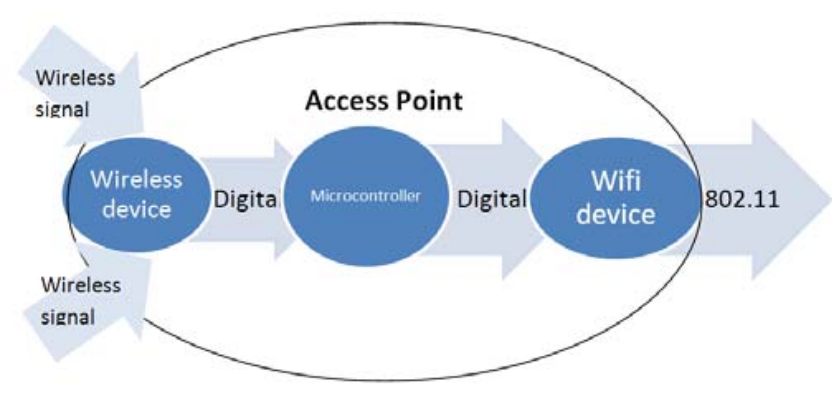

Figure6: Schematic diagram of access point.

Access point has identical design to the end device. The only difference is that it does not accept input from an accelerometer; instead, its input comes from a wireless device that received its data from radio frequency.

The microcontroller in AP can further calculate the input to share the load with ED if required. The output from the microcontroller will then be broadcasted wirelessly. Figure 6 shows the Wi-Fi connection to be 802.11 because the system in $\mathrm{OWN}$ in Figure 3 connects to a router.

\section{3) Network overview}

Figure 7, illustrates the configuration of the combined connection for both ED and AP.

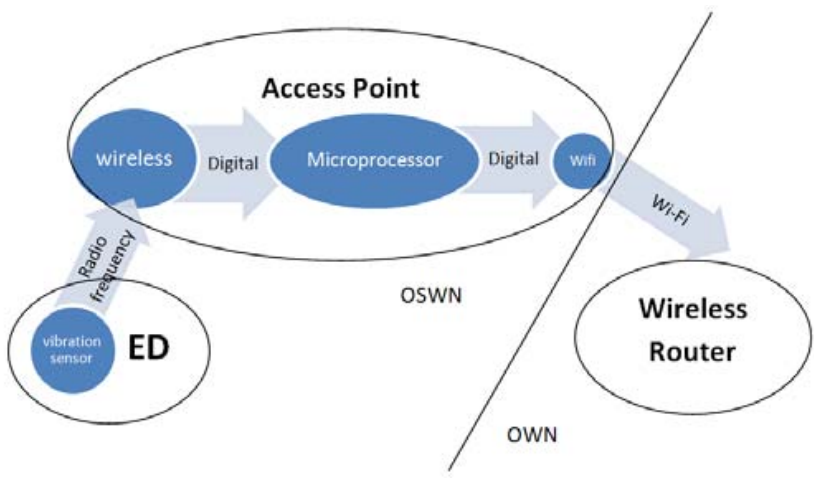

Figure7: Schematic diagram of overall network

OSWN is a short range wireless network and OWN is covered a further distance. The reason why OSWN operated in radio frequency is because it does not require complex protocol in its connection. Unlike radio frequency, there are a few handshaking protocols for the Wi-Fi connection with the wireless router. There is always the security part where credentials are required to request connection with the router. Wireless router is not part of evaluation in the project since it is assumed to be a general wireless router.

\section{SENSOR DESIGN}

Sensor design is a mock up design based on the conceptual design. This project will only focus on the vibration sensor design as the rest of the hardware is basically off the shelves. Using the conceptual system, the sensor can be explained by hardware and software field.

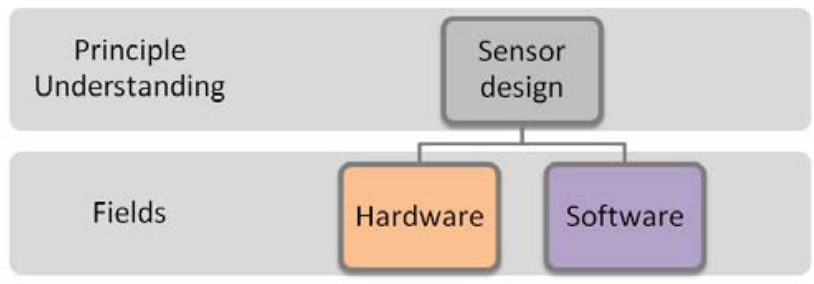

Figure8: Draft design section.

Figure 8 shows the two fields in the sensor. Both fields are basically self- explanatory.

\section{A. Hardware}

The hardware field for the sensor will obviously be similar to Figure 5. There are 3 major components.

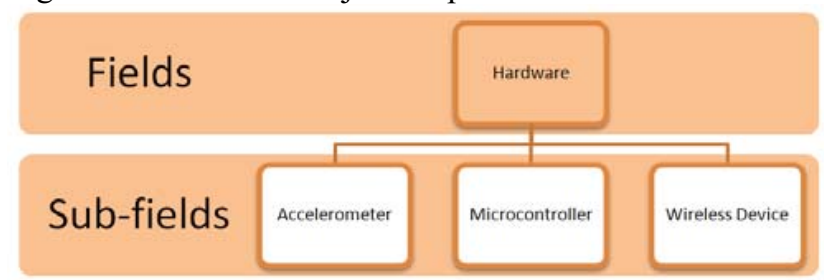

Figure 9: Hardware design section. 
Figure 9 is just a recap of the 3 components that are contained in the end device. The purpose of the hardware has already been described in chapter 3.1.2.1 to 3.1.2.3.

Since there are varies manufacturer that produces similar product, the list needs to be narrowed to the product that fits the requirement analysis of the product in mind.

\section{1) Accelerometer requirement analysis}

The accelerometer requirement analysis shows the minimal required features or operation range for the accelerometer. These expectations of basic feature below are sorted in descending order for filtering the selecting option from border available manufactures.

- Digital

- 3-axis

- Frequency response: $10 \mathrm{~Hz}$ to $1000 \mathrm{~Hz}$

- Measurement range: $\pm 15 \mathrm{~g}$ (at least)

- Low voltage consumption (less than $100 \mathrm{mV}$ )

- Small dimension (not larger than $20 \mathrm{~mm}$ X 20mm)

\section{2) Microcontroller requirement analysis}

The hardware compatibility is important in design phase to determine the product selection. This is because the physical connection between each electronic device has its particular communication port, for instant, I2C, SPI, UART or USART etc. Since the microcontroller is the center of the device, it needs to be compatible with both the accelerometer and the wireless transmitter.

- Digital

- Compatible with accelerometer connection

- Compatible with Wi-Fi device connection

- Easily programmable

- Hardware interrupts connection

- Low voltage consumption

- Small dimension

\section{3) Wireless connection requirement analysis}

The wireless connection in the whole system includes the OWN and OSWN as mentioned in Figure 4. As explained in 3.1.3, the wireless bandwidth used between the AP and the router must be 802.11 . However, there are a few connections available in this protocol. All the available wireless protocol will be listed in the next chapter.

The next wireless connection is the one between the ED and AP device. The wireless connection will be radio frequency as stated in chapter 3.1.4. Radio frequency also has multiple frequency bands. Some frequency bands are prohibited as it belongs to channel such as police communication channels. The option will be explored in detail later in the report [11].
B. Software

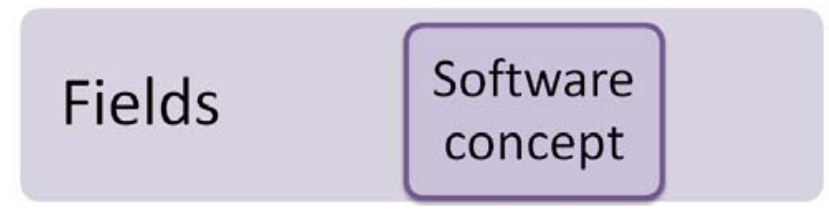

Figure10: Software design section.

Software phase is primarily design for hardware initialization and theoretical equation manipulation process. Software phase is divided into three parts as ED, AP and PC. Software design has the following tasks for each part in achieving the requirement of hardware health monitoring system [12].

- $\quad$ ED (End Device)

- Detect the vibration signal

- Manipulate the input signal

- Obtain result

- Check wireless connection status

- Handshake with wireless network

- Send data

O Check the result for correction

- $\quad$ AP (Access Point)

- Search for Wi-Fi

- Connect to router wait for PC to ask AP to work

- Connect to ED

- Get the signal from ED

- Further manipulate the signal

- Obtain result

○ Send data

- Check the result for correction

- $\quad$ PC (Client Software)

○ Wait for user to press connect.

- Individual or group ED selection

- PC check connection with AP through router

- Handshake with network

○ Draw a chart

- Simulate vibration profile

\section{Testing}

The testing model will be based on a free-free beam platform for the prototype sensor. 


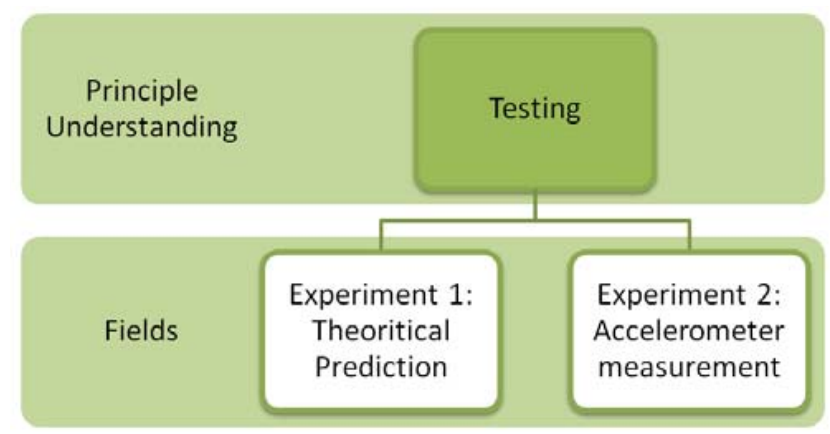

Figure11: Testing method section.

Figure 11 present two experiments that will examine the response of a free-free beam. First experiment is a virtual experiment that is performed using Matlab. Matlab will produce a set of theoretical data. This approach calculates and simulates the vibration of the beam under ideal condition.

The second part of testing method is performed by using WiVi vibration sensor to detect the vibration behavior. Both the results will be compared in order to assure the performance of WiVi vibration sensor.

\begin{tabular}{|c|c|}
\hline $\begin{array}{c}\text { Number of natural } \\
\text { frequency }\end{array}$ & Frequency $(\mathrm{Hz})$ \\
\hline $1^{\text {st }}$ & $17.512 \times 10^{3}$ \\
\hline $2^{\text {nd }}$ & $17.542 \times 10^{3}$ \\
\hline $3^{\text {rd }}$ & $17.560 \times 10^{3}$ \\
\hline
\end{tabular}

Figure 13: Out comes by using Matlab

\section{Future work}

This section is mentioned the work that is unfinished due to the limiting time frame. Nevertheless, the section is following by some expectations field which has a lot of potential spatial to improve on this particular project. Mainly, this paper is under a conceptual stage with design phase that contributes to the WiVi sensor design.

\section{CONCLUSION}

The objective of this research is to propose a new design to replace the SmartVib sensor. The new design is comprised of the latest technology. A prototype was unable to be actualized due to insufficient time. However, the conceptual design is completed.

The design has achieved digital transference, wireless communication, and substitutable modules.

\section{REFERENCES}

[1] MOORE'S LAW. intel.

http://www.intel.com/about/companyinfo/museum/exhibits/moore.ht $\mathrm{m}$ (accessed October 10, 2009).

[2] Howard, I. 2009. Lecture 7: Instruction to Finite Element Modeling. I2C-bus specification and user manual. Image. 2007. http://www.nxp.com/documents/user_manual/UM10204.pdf (accessed November 8, 2009).

[3] Akhondi, M., Talevski, A., Carlsen, S. and Petersen, S. 2010 Applications of wireless sensor networks for oil, gas and resources industries.CS Digital Library journal.

http://www.computer.org/portal/web/csdl/doi/10.1109/AINA.2010.18 (accessed December 7, 2010).

[4] Eren, H. 2006. Wireless Sensors and instruments. NW: Taylor \& Francis. Fast Fourier Transform. 1999. http://mathworld.wolfram.com/FastFourierTransform.html (accessed August 22, 2009).

[5] University of Rhode Island Department of Electrical and Computer Engineering ELE 436: Communication Systems. n.d. FFT tutorial. http://www.ele.uri.edu/ hansenj/projects/ele436/fft.pdf (accessed August 22, 2009).

[6] Inman, D. 2007. Engineering Vibration. New Jersey: Pearson education.

[7] The Weak Dirichlet Condition for the Fourier Series. 2010. Ricardo Radaelli-Sanchez. http://cnx.org/content $/ \mathrm{m} 10089 /$ latest/. (accessed October 10, 2009).

[8] Mechanics-of-MaterialsFall1999. Image. 1999. http://ocw.mit.edu/NR/rdonlyres/Materials-Science-andEngineering/3-11Mechanics-of-MaterialsFall1999/698ECA59-8F0046CC-96A1-B9365CBD63E3/0/props.pdf (accessed December 22. 2009).

[9] Accelerometer selection based on applications. 1999. http://www.hightech-pr.com/endevco/2006_08/2006_08_f4.pdf (accessed October 10, 2009).

[10] Trends in accelerometer design for military and aerospace application.Image.2009. http://archives.sensorsmag.com/articles/0399/0399_44/index.htm (accessed July 15, 2009).

[11] EE359: Wireless communications. Image. n.d. http://www.stanford.edu/class/ee359/flecture/lecture1.pdf (accessed October 10, 2009).

[12] Eren, H. 2006. Wireless Sensors and instruments. NW: Taylor \& Francis.FastFourierTransform.1999. http://mathworld.wolfram.com/FastFourierTransform.html (accessed August 22, 2009). 